Europhysics Letters

PREPRINT

\title{
Statistics of precursors to fingering processes
}

\author{
Patrick Grosfils ${ }^{1}$ and Jean Pierre Boon ${ }^{1}$ \\ 1 Physics Department CP 231, Université Libre de Bruxelles, \\ 1050 - Bruxelles, Belgium (E-mail: pgrosfi@ulb.ac.be ; jpboon@ulb.ac.be)
}

PACS. 05.90.+m - Statistical physics.

PACS. 05.40.-a - Fluctuation phenomena.

PACS. 05.10.Gg - Stochastic analysis (Fokker-Planck equation).

\begin{abstract}
We present an analysis of the statistical properties of hydrodynamic field fluctuations which reveal the existence of precursors to fingering processes. These precursors are found to exhibit power law distributions, and these power laws are shown to follow from spatial $q$-Gaussian structures which are solutions to the generalized non-linear diffusion equation.
\end{abstract}

Over the past fifteen years, a nonextensive variant to the Boltzmann-Gibbs formulation of Statistical Mechanics has been developed for the analysis of systems away from the equilibrium state [1] characterized by the appearance of non-exponential distributions and power laws. For instance, for diffusion type processes, non-exponential distributions are obtained from generalized Fokker-Planck type equations, and it has been shown, in a generic manner from a generalization of classical linear response theory, that such distributions may arise from first-principle considerations [2]. The goal of this letter is (i) to show that in fingering phenomena, patterns are preceded by precursor processes, and (ii) to present an analysis of the statistical properties of hydrodynamic field fluctuations in these precursors; we find power law distributions which are shown to follow from the solution of the generalized non-linear diffusion equation. Although we do not use nonextensive statistical mechanics to explain our results, we find mathematical similarities which suggest a possible connection as discussed in the concluding paragraph.

The phenomena investigated here arise before the onset of fingering, a generic phenomenon that results from the destabilization of the interface between two fluids with different mobilities in systems such as a shallow layer or a porous medium, when the fluid with highest mobility is forced through the medium filled with the other fluid. Here before means that the constrained fluid is in a state where no fingering pattern is as yet visible, but where hydrodynamic field fluctuations are enhanced as precursors to the onset of fingering. The analytical form of the statistical properties of these precursors are compatible with the solution of the generalized diffusion equation [2] which has formally the same structure as the "porous media equation" [3], but where the diffusion coefficient depends on the solution of the equation. This leads to the fact that the diffusion process is classical in the sense that there is linear scaling with time, but the solutions are not Gaussian: they have the canonical $q$-exponential form [1].

To date - at least to the best of our knowledge - such precursor properties have not yet been obtained from laboratory measurements. Here we use two methods: (1) a mesoscopic

(C) EDP Sciences 


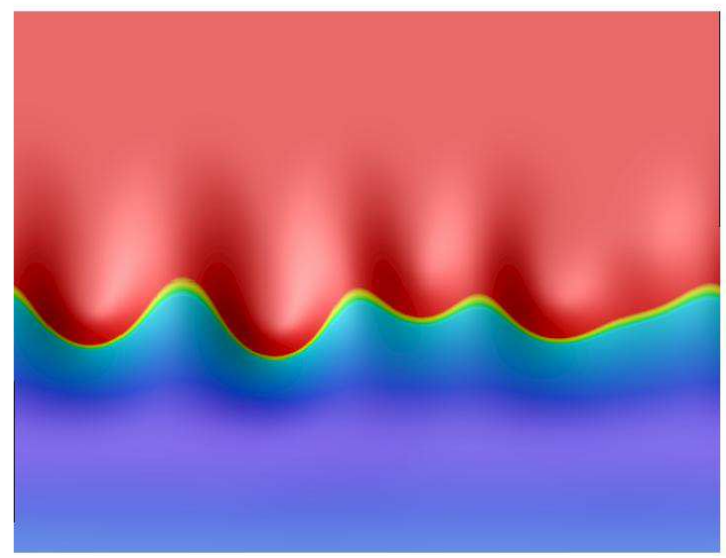

Fig. 1 - Illustration of lattice Boltzmann (LBGK) simulation of viscous fingering (flow direction downwards). The mixing length between the two fluids is clearly identified by the shadow zone around the concentration $=0.5$ contour line (color code indicating the concentration of the invading fluid from red $\left(C_{1}=1\right)$ to blue $\left.\left(C_{1}=0\right)\right)$.

approach, the lattice Boltzmann simulation method [4], which is based on a kinetic theoretical analysis where the macroscopic description is not pre-established, and (2) a phenomenological approach, Darcy's law [5] which is solved numerically. Our results show that (i) the system's non-linear response to the driving force produces dynamical behavior in accord with the generalized diffusion equation while no such phenomenological prescription was injected a priori, (ii) the statistics of the hydrodynamic fluctuations reveal the existence of precursor processes before fingering patterns become visible, (iii) the distribution of these field fluctuations are given by power laws (with an exponent $|q|<1$ ), and (iv) these power laws follow from space integration of two-dimensional $q$-Gaussian structures.

These structures form a landscape of alternating upward and downward $q$-Gaussian "blobs" (see Fig.2 below) which are precursors to the fingers, and are reminiscent of vortices with alternating parities in $2-d$ turbulence $\left(^{1}\right)$. For instance, experiments in turbulent CouetteTaylor flow [6] have shown that data obtained from quantities extracted from velocity field measurements exhibit $q$-exponential distributions which have been analyzed with theoretical arguments based on nonextensive statistical mechanics [7]. Other physical systems have been shown to exhibit similar type distributions interpreted as a consequence of nonextensivity [1] or superstatistics [8]. We suggest that in the early stage of the fingering process, the signature of nonextensivity can be found in the recasting of interacting Gaussian structures into a sum of independent $q$-Gaussian blobs.

We consider a two miscible fluids system confined between two plates with narrow gap, a configuration known as the Hele-Shaw cell [9]. The Hele-Shaw geometry is intrinsically threedimensional, but the effective destabilization of the flow can be described in the 2- $d$ plane, given that the flow has a Poiseuille profile in the third dimension. So Hele-Shaw flow can be simulated with the two-dimensional LBGK equation, the Bhatnagar-Gross-Krook single

$\left({ }^{1}\right)$ Here the Reynolds number has low value, but the relevant control parameter, the Péclet number, is high. 




Fig. 2 - Concentration fluctuations field showing landscape of $q$-Gaussian "hills and wells" (flow direction South-East); simulation data with concentration contour lines, color code indicating highest positive values (red) to largest negative values (magenta) of $c$.

relaxation version of the lattice Boltzmann equation [4]. One emulates 3- $d$ flow by introducing a drag term, thereby simulating a system with a virtual cell gap in the third dimension: the drag enters the LBGK equation as a damping term. The simulation method has been described elsewhere [10]: essentially the system consists of a $2-d$ box with vertical length $L_{y}=2048$ nodes, and horizontal width $L_{x}=1024$ nodes, with horizontal periodic boundary conditions; initially the box is filled with fluid 2 which is then displaced by fluid 1 injected uniformly from the top of the box. Fluid 1 (with damping coefficient $\beta_{1}<\beta_{2}$ ) invades the system through a constant force applied along the $y$-direction, which produces a flow with Péclet number $P e \simeq 200$.

The initial concentration profile is a step function, which because of mutual diffusion, develops into an erfc $y$, on top of which white noise is imposed (along the $x$-direction) to trigger the instability. When the instability develops, it produces a moving fingering pattern as illustrated in Fig.1. Here we consider the early stage where fingers are not yet visible (they have not developed as in Fig.1 shown here for the purpose of illustration), just below the dynamical transition where the exponent $\mu$ of the growth of the mixing length of the interfacial zone, $L_{m i x} \propto t^{\mu}$, changes from $\mu=1 / 2$ (diffusive regime) to $\mu \simeq 2.3$ (see Fig. 3 in [10]). In this early time regime, the flow produces local concentration gradients which induce mobility fluctuations thereby triggering vorticity fluctuations.

We measured the following local fluctuations:

- concentration field (fluid 1): $c(x, y)=C(x, y)-\overline{C(x, y)}$;

- transverse velocity field: $v_{x}(x, y)=V_{x}(x, y)-\overline{V_{x}(x, y)}$

- longitudinal velocity field: $v_{y}(x, y)=V_{y}(x, y)-\overline{V_{y}(x, y)}$;

- and vorticity field: $\omega(x, y)=\partial_{x} v_{y}(x, y)-\partial_{y} v_{x}(x, y)$,

where the overline denotes average along the $x$-direction. The concentration field in Fig.2 shows a "landscape" of alternating hills and wells. In each blob, the concentration field exhibits a two-dimensional $q$-Gaussian profile as illustrated in the left panel of Fig.3 obtained by a section plane cut through the extrema in Fig.2 parallel to the $x$-axis. The corresponding concentration distribution, $P(c)$, is obtained by taking the values of $c(x, y)$ measured over all 

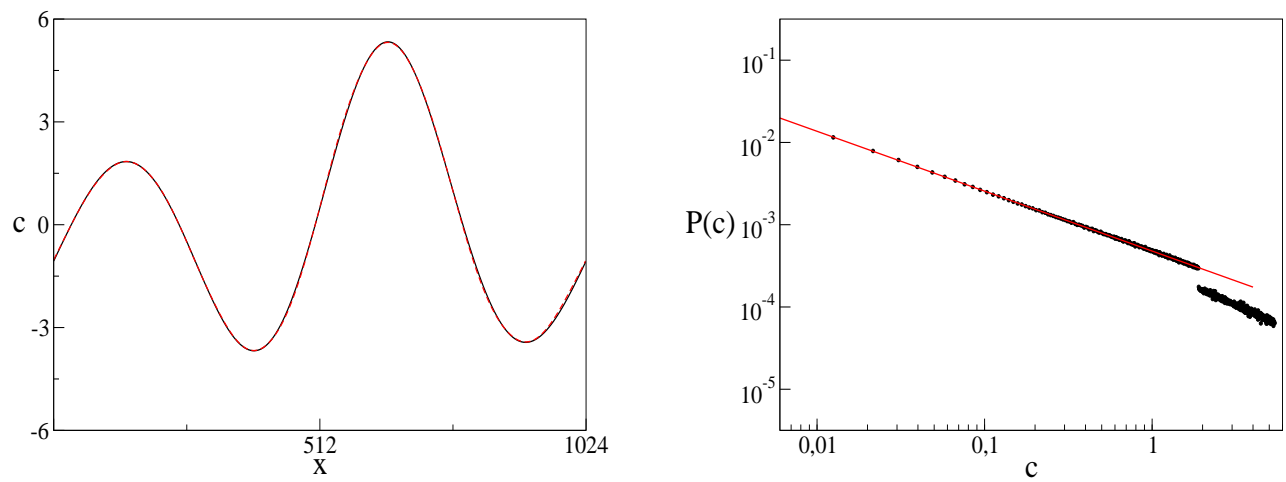

Fig. 3 - (a) Left panel: Concentration fluctuations profile (solid line) obtained from the simulation data by a section plane cut through the hills and wells in Fig.2; analytical function Eq.(2) (dashed line): the convex and concave curves are connected by summation of four $q$-Gaussians; (b) Right panel : Concentration fluctuations distribution (dots = data) with power law (solid line): $P(c)=$ $A|c|^{-q}$ with $q=0.73$.

space, and is shown to follow a power law, $P(c) \propto|c|^{-q}$ with $q=0.73$ (see right panel of Fig.3). Note that the power law exponent stays the same over the entire range of the distribution. The other local fields exhibit similar behavior and the corresponding distributions $P\left(v_{x}\right), P\left(v_{y}\right)$ and $P(\omega)$ all show power laws (with an exponent $<1$ ) which follow from space integration (see below).

Processes stemming from the generalized diffusion equation [2] (or from the generalized Fokker-Planck equation provided some conditions are met for the drift and dissipative functions [11-13]) can produce solutions of the $q$-exponential type. In the present simulations, we observe that the concentration field fluctuations exhibit a $q$-Gaussian profile - while the system is still in the diffusive regime $\left(\sim t^{1 / 2}\right)$ - which suggests that the concentration $c(r, t)$ is governed by the generalized $d$-dimensional non-linear diffusion equation [2] $\left({ }^{2}\right)$

$$
\frac{\partial c(r, t)}{\partial t}=\frac{1}{r^{d-1}} \frac{\partial}{\partial r}\left[r^{d-1} D(r) \frac{\partial}{\partial r} c^{\alpha}(r, t)\right] .
$$

A general solution to Eq.(11) is indeed a $q$-exponential of the form [2]

$$
c(r)=c_{0} e_{q}^{-\gamma_{q}|r|^{2}} \equiv c_{0}\left[1-(1-q) \gamma_{q}|r|^{2}\right]^{\frac{1}{1-q}},
$$

where $c_{0}$ and $\gamma_{q}$ are time-dependent quantities, and $q+\alpha=2$. This solution (taken at fixed $t$ ) is used to perform a fit to the simulation data, and the concentration profile found from the simulation results has exactly the form of (2) as shown in Fig.3a.

On the other hand, the velocity field is related to the concentration through Darcy's law (see e.g. [9]) which can be written for the stream function $\psi$ (in two dimensions) as

$$
\left(\kappa_{x} \frac{\partial}{\partial x}+\kappa_{y} \frac{\partial}{\partial y}\right) \psi(x, y)=\frac{1}{R(x, y)} \nabla^{2} \psi(x, y)
$$

$\left({ }^{2}\right)$ On the r.h.s. of 11 one can rewrite formally $D \partial_{r} c^{\alpha}$ as $D_{\alpha} \partial_{r} c$, where $D_{\alpha}=\alpha D c^{\alpha-1}$. 

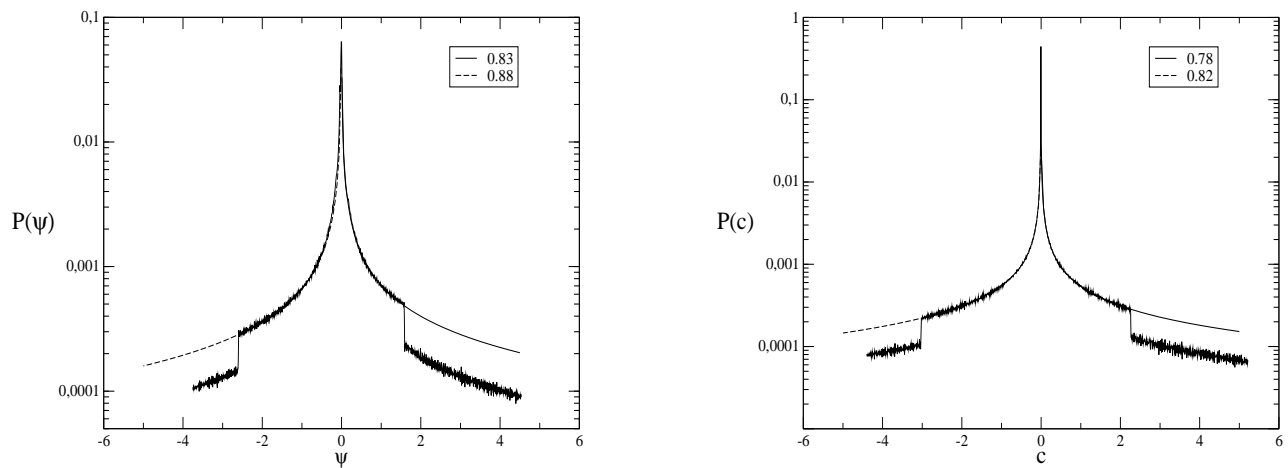

Fig. 4 - (a) Left panel: Stream function distribution (dots = data from numerical solution of Eq.(3)) with power law (solid and dashed lines) $P(\psi) \propto|\psi|^{-q^{\prime}}$ (8) with $q^{\prime}=0.83$ for $|\psi>0|$, and $q^{\prime}=0.88$ for $|\psi<0|$. (b) Right panel: concentration field distribution (dots = data from numerical solution of classical advection-diffusion equation coupled to Eq. (3)) with power law (solid and dashed lines) $P(c) \propto|c|^{-q}$ with $q=0.78$ for $|c>0|$, and $q=0.82$ for $|c<0|$.

with $\kappa_{x}=\partial c / \partial x$, and $R=\partial \ln \beta / \partial c$, where $\beta$ is the damping function which, in the fingering simulations, controls the drag and depends on the concentration $c$. Equation (3), coupled with the classical advection-diffusion equation for $c$, was solved numerically under conditions similar to those used for the lattice Boltzmann simulations. For both the concentration field and the stream function, we obtain $q$-Gaussians in agreement with the data obtained from the LBGK simulation results; in particular, for the stream function, we have

$$
\psi(r)=\psi_{0}\left[1-\left(1-q^{\prime}\right) \phi_{q^{\prime}} r^{2}\right]^{\frac{1}{1-q^{\prime}}},
$$

where $\psi_{0}$ is a normalization constant

We now show that the distribution which follows from the $q$-Gaussian spatial profile is a power law. Generalizing the $q$-exponential in (44) to an arbitrary power $\lambda$ of the radial variable $r$, we have

$$
\psi(r)=e_{q}^{-\phi_{q^{\prime}} r^{\lambda}} \equiv\left[1-\left(1-q^{\prime}\right) \phi_{q^{\prime}} r^{\lambda}\right]^{\frac{1}{1-q^{\prime}}} .
$$

(for simplicity we have incorporated the normalization into $\psi$ ), and the corresponding probability distribution function $P(\psi)$ reads (in $d$ dimensions)

$$
P(\psi)=\varpi_{d} \int_{0}^{\infty} r^{d-1}|d r| \delta(\psi(r)-\psi),
$$

where $\varpi_{d}$ is an integration constant. Then (5) is inverted to give $r^{\lambda}=\frac{1-\psi^{1-q^{\prime}}}{\phi_{q^{\prime}}\left(1-q^{\prime}\right)}=-\frac{1}{\phi_{q^{\prime}}} \ln _{q^{\prime}} \psi$, which is used in (6) to obtain

$$
P(\psi)=\varpi_{d} \frac{|r|^{d-\lambda} \psi^{-q^{\prime}}}{\lambda \phi_{q^{\prime}}}=\frac{\varpi_{d}}{\lambda \phi_{q^{\prime}}^{d / \lambda}}\left[-\ln _{q^{\prime}} \psi\right]^{\frac{d-\lambda}{\lambda}} \psi^{-q^{\prime}}
$$



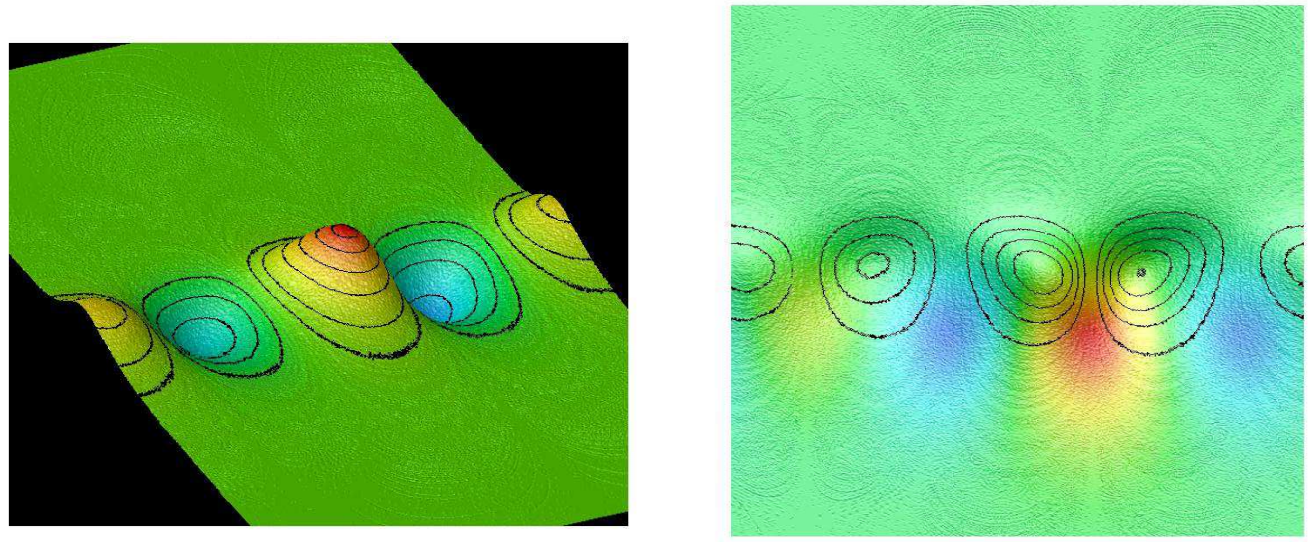

Fig. 5 - (a) Left panel: Vorticity field showing landscape of $q$-Gaussian "hills and wells" (flow direction South-East); simulation data with vorticity contour lines, color code indicating highest positive values (red) to largest negative values (blue) of $\omega$. (b) Right panel: Contour lines of vorticity field (simulation data) superimposed on color concentration fluctuations field (simulation data); color code indicates highest positive values (red) to largest negative values (blue) of $c$. The inflection line of the concentration field $(C \simeq 0.5)$ is about two thirds down from top of picture (flow direction downwards).

For two-dimensional systems $(d=2)$ with $q$-Gaussian spatial profile $(\lambda=2)$, we obtain the power law

$$
P(\psi)=\frac{\varpi_{2}}{2 \phi_{q^{\prime}}} \psi^{-q^{\prime}},
$$

which is exactly what we find for the stream function distribution shown in Fig.4a where a fit to the simulation data gives an exponent $q<1$ (indicating statistical importance of large values of the stream). The distribution $P(c)$ (Fig.4b) is obtained along the same lines and yields the value $q \simeq 0.80 \pm 0.05$ to be compared with the value $0.73 \pm 0.05$ obtained from the lattice Boltzmann simulations (see Fig.3). Singularities (elliptic singularities [14]) in the distribution function occur where the field has extrema (corresponding to the maximum of the absolute value of the blobs). According to Eq.(11), the value of $q$ obtained from the concentration profile and distribution data yields $\alpha \simeq 1.25$ in the diffusion term of Eq. (11), thus an effective diffusion coefficient $D_{\alpha} \propto c^{0.25}$ (see footnote (2)). These results indicate that the generalized diffusion equation governs effective transport in fingering phenomena.

We also measured the vorticity field which, as shown in Fig.5a, exhibits a pattern of alternating vortices which can be viewed as a landscape of hills and wells of radial $q$-Gaussians distributed along the concentration profile of the interfacial zone. Figure 5b shows the respective locations of the vorticity field (contour lines) and concentration field (color coded).

Two questions arise from the present results: (i) what is the physical picture of the precursors dynamics? and (ii) given that many systems exhibiting similar features have been analyzed with the nonextensive approach [15], we pose the reciprocal question: can one suggest a physical interpretation of nonextensivity from the structure of the precursors?

(i) We observe that, before any fingering pattern becomes visible, the driving force triggers pre-transitional fluctuations in the concentration field in the form of blobs organized spa- 
tially according to the wavelength of the forthcoming fingers (Figs.2 and 3a). Concentration fluctuations modify locally the $C$-dependent drag coefficient thereby inducing shear. As a consequence, the initially uniform velocity field is perturbed, and the resulting velocity fluctuations produce vortices with alternating parities and whose spatial sequence matches (with phase shift) the concentration fluctuation structures (Fig.5). The configuration shown in Fig.5b is reminiscent of the fingering vortices depicted in a schematic in [16] indicating that the mechanism described in [16] is already present in the precursors.

(ii) The system studied here is representative of a generic class of driven nonequilibrium systems with spatial fluctuations, where $q$-exponentials and power law distributions are the signature of long-range interactions, and whose dynamical behavior is governed by advectiondiffusion equations. What we have shown is that during the onset of fingering, one can identify precursors which exhibit features with mathematical properties typically encountered in nonextensive statistics. This similarity suggests a possible interpretation of the mechanism of nonextensivity. The driving force produces a spatial sequence of alternating structures, which, if they were independent, would exhibit ordinary Gaussian profile originating from local diffusion centers ( $\delta$-functions), and would be described by classical diffusion. However, when growing, these Gaussians overlap, and what the analysis shows is that the structure formed by these overlapping Gaussians can be cast into a sum of scale invariant $q$-Gaussians with finite support $(q<1)$, i.e. these $q$-Gaussians are independent as corroborated by the fact that the power law distribution is shown (see Eq.(8) ) to follow from space integration of a single $q$-exponential.

This work was supported by a grant from the European Space Agency and PRODEX (Belgium) under contract ESA/14556/00/NL/SFe(IC).

\section{REFERENCES}

[1] C. Tsallis, Physica D, 193 (2004) 3, and references therein.

[2] J.F. Lutsko And J.P. Boon, Europhys. Lett., 71 (2005) 906

[3] A. Compte and D. Jou, J. Phys. A, 29 (1996) 4321.

[4] S. Succi, The Lattice Boltzmann Equation for Fluid Dynamics and Beyond (Clarendon Press, Oxford) 2001.

[5] See E.G. J. Bear, Dynamics of Fluids in Porous Media (Dover, New York) 1988.

[6] C. Beck, G.S. Lewis, and H. L. Swinney, Phys. Rev. E, 63 (2001) 035303(R).

[7] C. Beck, Phys. Rev. Lett., 87 (2001) 180601.

[8] C. Beck and E.G.D. Cohen, Physica, 322A (2003) 267; C. Beck, Superstatistics: Recent developments and applications, (2005) arXiv:cond-mat/0502306.

[9] G.M. Homsy, Ann. Rev. Fluid Mech., 19 (1987) 271.

[10] P. Grosfils, J.P. Boon, J. Chin, and E.S. Boek, Phil. Trans. Royal Soc., 362 (2004) 1723.

[11] C. Tsallis and D.J. Bukman, Phys. Rev. E, 54 (1996) R2197.

[12] L. Borland, Phys. Rev. E, 57 (1998) 6634.

[13] I.T. Pedron, R.S. Mendes, L.C. Malacarne, and E.K. Lenzi, Phys. Rev. E, 65 (2002) 041108.

[14] G.M. Zaslavsky, R.Z. Sagdeev, D.A. Usikov, and A.A. Chernikov, Weak Chaos and Quasi-Regular Patterns (Cambridge University Press, Cambridge, 1991; sec. 7.4).

[15] SEE E.G., EuroPhysics News, 36/6 (2005) 183-231.

[16] A. De Wit and G. M. Homsy, J. Chem. Phys., 110 (1999) 8663; see Fig.6 and section V.B. 